\title{
Estudio de un cálculo intestinal en un paciente con adenocarcinoma de colón. ¿es similar a los cálculos renales?
}

\author{
M.L. Traba Villameytide*, JA Orts Costa**, M. Morell*** \\ *Laboratorio de Bioquímica. Sección de Fisiopatología Ósea. *Fundación Jiménez Díaz-UTE. Madrid. \\ **Área de Diagnóstico Biológico. ${ }^{* * *}$ Servicio de Anatomía Patológica. ${ }^{* * * * * * H o s p i t a l ~ d e ~ l a ~ R i b e r a . ~ A l z i r a, ~ V a l e n c i a ~}$
}

Actas Urol Esp 2006; 30 (2): 206-214

\section{RESUMEN}

\section{ESTUDIO DE UN CÁLCULO INTESTINAL EN UN PACIENTE CON ADENOCARCINOMA DE COLÓN.} ¿ES SIMILAR A LOS CÁLCULOS RENALES?.

Este trabajo muestra el estudio realizado a enterolitos intestinales procedentes de un paciente de 91 años que padecía una enterolitiasis múltiple confirmada por estudio radiológico abdominal y TAC, mostrando cálculos en el tracto intestinal, renal y biliar. Además esta enterolitiasis estaba asociada a un adenocarcinoma de colon. Los enterolitos analizados proceden de una intervención quirúrgica en la que se practicó una hemicolectomía derecha.

Los enterolitos se sometieron a un análisis por espectrometría de infrarrojos (IR) observándose un espectro de carbonato apatita no-estequiométrica, tipo whitloquita, posiblemente con materia orgánica. Con el fin de estudiar el posible contenido de diversos elementos químicos, se practicó un análisis por espectrometría de emisión atómica encontrándose, fundamentalmente, los iones $\mathrm{Ca}, \mathrm{Mg}, \mathrm{K}$, Na y K (del orden de mg/100 mg de cálculo) y Zn, $\mathrm{Ba}, \mathrm{Mn}, \mathrm{Fe}, \mathrm{Cu}, \mathrm{Si}$, Ti y Br en menor proporción (del orden de $\mu \mathrm{g} / 100$ mg de cálculo). Dada la morfología del cálculo y su espectro de IR (carbonato apatita no estequiométrica) se determinó la posible presencia de porfirinas por cromatografía líquida de alta resolución (HPLC) encontrándose, fundamentalmente, coproporfirina ( $\mu \mathrm{g} / \mathrm{g}$ de cálculo) y en menor proporción uroporfirina, protoporfirina y hepta-carboxi porfirina. El estudio se completó con el análisis de los enterolitos mediante microscopía electrónica de barrido y EDX. El análisis por difracción de rayos $\mathrm{X}$ detectó la presencia de $\mathrm{CaP}_{4} \mathrm{O}_{11}$.

Los resultados obtenidos de los diferentes análisis muestran que la composición de los enterolitos es similar a la de los cálculos renales, aunque su morfología difiera de estos.

Palabras clave. Enterolitiasis. Carbonato apatita no-estequiométrica. Coproporfirina. Fosfato cálcico $\left(\mathrm{CaP}_{4} \mathrm{O}_{11}\right)$.

\section{ABSTRACT}

\section{STUDY OF A INTESTINAL ENTEROLITHS IN HUMAN PATIENT WITH COLON ADENOCARCINOME.} IS IT SIMILAR TO RENAL CALCULI?

This work shows the study performance to intestinal enterolithis from a 91 year old patient with multiple enterolithiasis confirmed by abdominal X-ray and TAC analyses showing the presence of intestinal, renal and bile stones. This enterolithis is associated with colon adenocarcinoma.

The enteroliths were obtained by hemicolectomia and were analyzed by infrared spectroscopy (IR), giving nonstoichiometry carbonate apatite whitloquite-like with, possibly, organic material. By atomic emission spectroscopy we found $\mathrm{Ca}, \mathrm{Mg}, \mathrm{K}$, Na y K (mg/100 mg of calculi) and $\mathrm{Zn}, \mathrm{Ba}, \mathrm{Mn}, \mathrm{Fe}, \mathrm{Cu}, \mathrm{Si}$, Ti and $\mathrm{Br}$ in minor proportion ( $\mu \mathrm{g} / 100 \mathrm{mg}$ of calculi). Because of calculi morphology and the IR spectra (non-stoichiometry carbonate apatite) we carried out analysis by high performance liquid chromatography (HPLC) and found coproporphyrin (about $\mu \mathrm{g} / \mathrm{g}$ of calculi) and uroporphyrin, protoporphyrin and heptacarboxy-porphyrin in minor extent. Calculi were also studied by scanning electronic microscopy and EDX and X-ray diffraction giving crystals of $\mathrm{CaP}_{4} \mathrm{O}_{11}$.

All these results show that intestinal enteroliths composition are similar to renal calculi although its morphology differs from renal calculi.

Keywords: Enterolithiasis. Carbonate apatite whitloquite-like. Coproporphyrin. Calcium phosphate $\left(\mathrm{CaP}_{4} \mathrm{O}_{11}\right)$. 
L a presencia de cálculos intestinales (coprolitos o enterolitos) en humanos es un hecho muy poco frecuente. No así en animales, como ocurre en equinos. Poco habitual es el caso de enterolitiasis múltiple con presencia de cálculos intestinales, renales y biliares. Estos datos confirmados por rayos $\mathrm{X}(\mathrm{RX})$ y tomografía axial computerizada (TAC) son de gran relevancia.

Este caso clínico tan peculiar nos llevó a realizar un estudio de los cálculos intestinales de forma exhaustiva. En el presente trabajo se ha realizado estudios de los cálculos mediante espectrometría infrarroja (IR), espectrofotometría de emisión atómica, cromatografía líquida de alta resolución (HPLC), microscopía electrónica de barrido-EDX y difracción de rayos $\mathrm{X}$.

\section{MATERIALES Y MÉTODOS}

Se han analizado enterolitos de intestino procedentes de un paciente de 91 años que padecía una enterolitiasis múltiple en intestino, vesícula biliar y tracto renal.

\section{Espectroscopia de absorción infrarroja}

Para obtener el espectro de absorción infrarroja (EIR) entre 4000 y $250 \mathrm{~cm}^{-1}$, se ha utilizado un espectrofotómetro Perkin-Elmer (modelo 457, España). Las células de absorción se han preparado mediante el procedimiento de los comprimidos de bromuro potásico.

\section{Determinación de elementos químicos}

La determinación de los diferentes elementos químicos presentes en las muestras se realizó con un espectrofotómetro de emisión atómica ICP-VISTA-PRO (Varian, Austria).

\section{Valoración de porfirinas}

Se ha realizado por análisis de cromatografía líquida de alta resolución (HPLC).

\section{Difracción de Rayos X}

La identificación de componentes cristalinos se ha realizado utilizando un difractómetro Philipsx Pert con radiación CuK. Las condiciones de extracción fueron de $40 \mathrm{Kv}$ y $50 \mathrm{~mA}$. Se utilizó un monocromador secundario de grafito y un contador miniproporcional. Los difractogramas se registraron con una velocidad de $0,02^{\circ} 2 \mathrm{O} / \mathrm{s}$.

\section{Microscopía electrónica}

Las muestras fueron procesadas por SEMBSE y análisis de EDS-EDX según el método desarrollado por Wierzchos y Acaso $^{1}$, con las siguientes modificaciones: las muestras fueron fijadas químicamente con $2,5 \%$ de glutaraldehído (v/v) en tampón fosfato $0.1 \mathrm{M}, \mathrm{pH}$ : 7.1. Todas las muestras fueron lavadas en filtros con agua esterilizada y fijadas posteriormente en tetróxido de osmio $\left(\mathrm{OsO}_{4}\right)$ al $2 \%(\mathrm{p} / \mathrm{v})$. Durante la hidratación con una serie de etanoles, algunas muestras fueron contrastadas con acetato de uranilo saturado con etanol al 70\% (v/v). Las muestras fueron embebidas en el medio de LR-White. Una vez polarizados, los bloques fueron cortados transversalmente usando una cuchilla de diamante y pulidos finamente con polvo de diamante. Las muestras fueron examinadas bajo microscopía electrónica de barrido con un detector que recoge los electrones retrodispersados (BSE-SEM) más un sistema microanalítico por energía dispersiva de rayos $\mathrm{X}$ (EDS, EDX). Secciones transversales de las muestras con las superficie pulida fueron examinadas usando un microscopio DSM 940 A Zeiss SEM equipado con un detector BSE y un sistema microanalítico Link ISIS EDS. Las condiciones de trabajo de EDS son: $0^{\circ}$ de ángulo de inclinación, $35^{\circ}$ de ángulo de salida/EDS, $15 \mathrm{Kv}$ de potencial de aceleración y de 6 a 25 (RDS) $\mathrm{mm}$ de distancia de trabajo con una corriente de 1-5 mA.

\section{RESULTADOS}

Dieciséis cálculos (coprolitos) fueron enviados al laboratorio de la Fundación Jiménez DíazUnilabs. La Figura 1 muestra las características morfológicas de estos enterolitos. Como puede apreciarse la mayoría son redondeados, de color marrón claro, con dibujos más oscuros en la superficie del cálculo. Al corte, mostraron un interior vacío y la cara cóncava estaba recubierta de unas proyecciones filamentosas. El peso total de los mismos fue de $4808.6 \mathrm{mg}$ y las dimensiones de $20 \times 15,20 \times 10,10 \times 6,9 \times 8 \ldots$

Las Figuras 2A y 2B corresponden a los espectros de infrarrojos (EIR) de los enterolitos en los que puede observarse una composición de carbonato apatita no estequiométrica y materia orgánica (comparar con el EIR de una carbonato apatita 


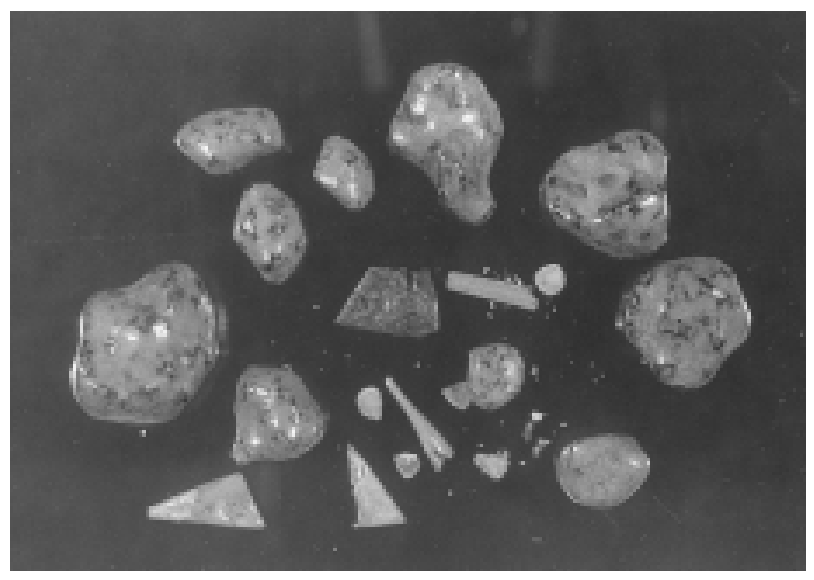

FIGURA 1. Morfologia de los enterolitos.

estequiométrica mostrado en la Figura 3. Las bandas encontradas en la región de $600 \mathrm{~cm}^{-1} \mathrm{se}$ asemejan a los que muestra la whitloquita (Fig. 4) por lo que parece tratarse de una carbonato apatita no estequiométrica tipo whitloquita.

La Tabla 1 recopila el contenido de los elementos químicos obtenidos por espectrofotometría de emisión atómica. Hay que destacar la alta concentración de $\mathrm{Ca}, \mathrm{P}$ y $\mathrm{Mg}$ (mg/100 mg de cálculo) mientras que hay otra serie de elementos presentes en menor proporción ( $\mu \mathrm{g} / 100 \mathrm{mg}$ de cálculo) entre los que destacan: $\mathrm{Zn}, \mathrm{Mn}, \mathrm{Fe}$ y Cu.
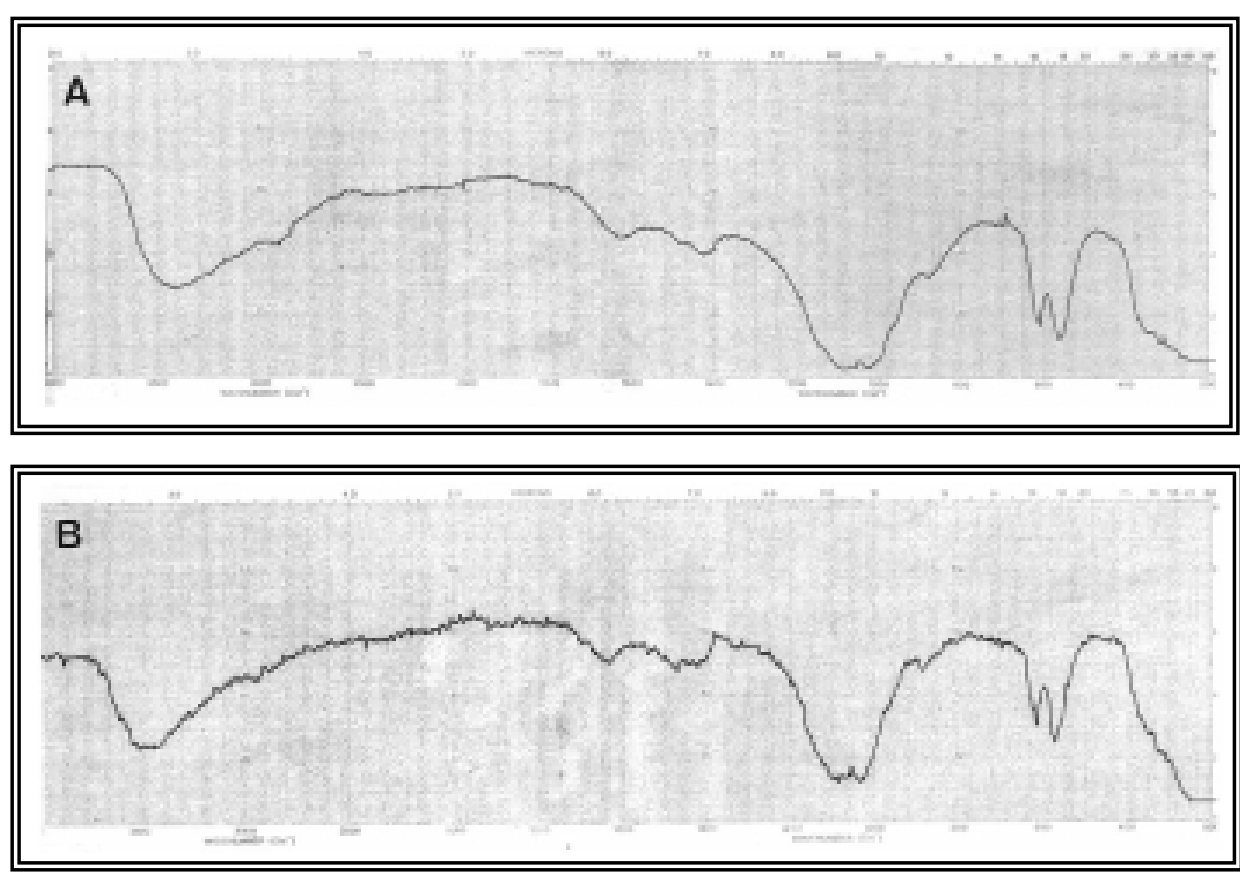

FIGURA 2 (A,B). Espectros de infrarrojos de los enterolitos.
Dadas las características del cálculo, especialmente su color y el EIR, se sospechó la existencia de porfirinas, hecho corroborado por cromatografía líquida de alta resolución (HPLC). Se encontró una alta concentración de coproporfirina $(3.4 \mu \mathrm{g} / \mathrm{g}$ de cálculo; ver Tabla 2) y en menor proporción uroporfirina, protoporfirina y heptacarboxilporfirina.

La Figura 5 muestra un difractograma procedente de un estudio realizado en un cálculo por difracción de rayos $\mathrm{X}$ que corresponde a un fosfato muy bien cristalizado, el $\mathrm{CaP}_{4} \mathrm{O}_{11}$.

Las imágenes recopiladas en la Figura 6 se obtuvieron con microscopía electrónica de barrido, mostrándose una serie de elementos poliédricos (Figura 6A) que podrían ser materia orgánica; un entramado de fibras (Fig. 6B); figuras poliédricas con zonas de material claro (lo que indicaría que están formados por materia de número atómico elevado) y otras zonas más oscuras (con número atómico menor) (Fig. 6C). La Figura 6D muestra una serie de puntos brillantes que podría ser materia cristalizada. La Figura 6E contiene una zona muy oscura y otra clara.

Las imágenes de la Figura 7 proceden del análisis por energía dispersiva de rayos $\mathrm{X}$ (lo que se denomina EDX) obteniéndose un "mapping" de un cálculo. En la Figura 7(A-C) pueden apreciarse esferulitos de carbonato apatita, representando un "mapping" que muestra los elementos $\mathrm{Mg}, \mathrm{P}$ y Ca. Sin embargo, en la Figura 7D, curiosamente se muestra que la zona correspondiente a las formas poliédricas $\mathrm{y}$ unas zonas más claras contienen $\mathrm{Mg}$ aunque su contenido de calcio desciende.

La Figura 8 muestra el estudio por EDX de las figuras poliédricas obteniéndose un contenido de $\mathrm{O}=72 \%, \mathrm{Na}=10,37 \%, \mathrm{Cl}=$ $7,55 \%$ y $\mathrm{Ca}=3,50 \%$. Además contiene otros elementos como $\mathrm{Mg}, \mathrm{P}$ y K en menor proporción. 


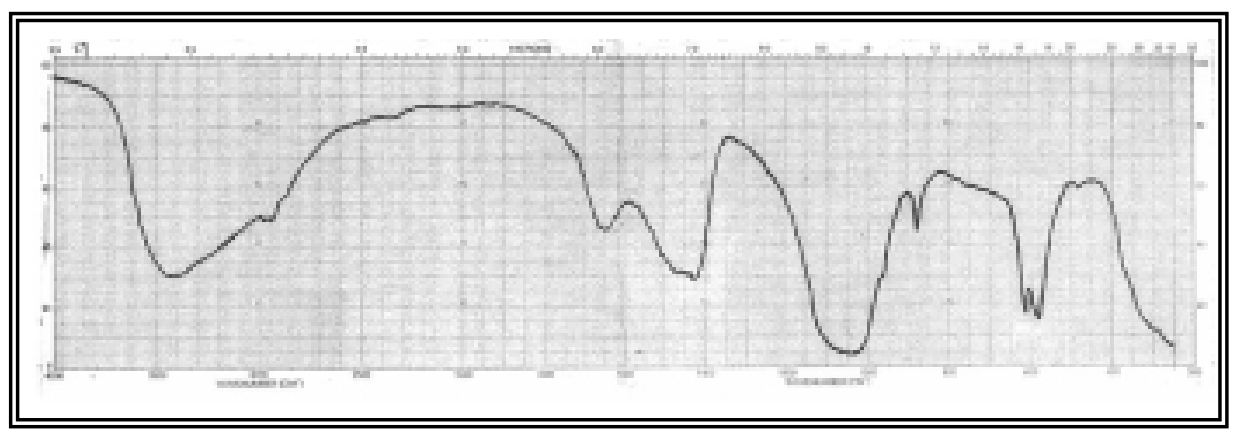

FIGURA 3. Espectro de infrarrojos de una carbonato apatita estequimétrica.

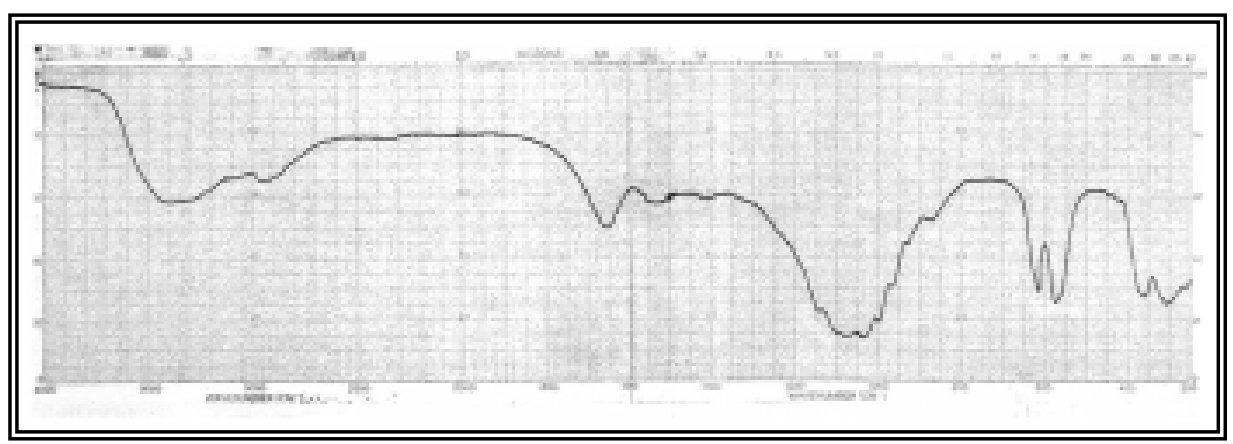

FIGURA 4. Espectro de infrarrojos de la whitloquita.

Tabla 1

Elementos obtenidos por espectrofotometría de emisión atómica

\begin{tabular}{cc}
\hline Elemento & $\mathbf{1 0 0} \mathbf{~ m g}$ de coprolito \\
\hline $\mathrm{Ca}$ & $19,4 \mathrm{mg}$ \\
$\mathrm{Mg}$ & $1,6 \mathrm{mg}$ \\
$\mathrm{K}$ & $0,12 \mathrm{mg}$ \\
$\mathrm{Na}$ & $0,44 \mathrm{mg}$ \\
$\mathrm{P}$ & $9,84 \mathrm{mg}$ \\
$\mathrm{Zn}$ & $75 \mu \mathrm{g}$ \\
$\mathrm{Ba}$ & $2,0 \mu \mathrm{g}$ \\
$\mathrm{Mn}$ & $27,8 \mu \mathrm{g}$ \\
$\mathrm{Fe}$ & $3,2 \mu \mathrm{g}$ \\
$\mathrm{Cu}$ & $3,2 \mu \mathrm{g}$ \\
$\mathrm{Si}$ & $3,2 \mu \mathrm{g}$ \\
$\mathrm{Ti}$ & Trazas \\
$\mathrm{Br}$ & Trazas \\
\hline
\end{tabular}

La Figura 9 muestra dos zonas bien definidas, una clara y otra oscura. El EDX de la zona clara (Fig. 9A) muestra un contenido de $\mathrm{O}=67,38 \%$, $\mathrm{Ca}=26,22 \%$ y $\mathrm{Mg}=4,73 \%$ y otros iones en menor concentración como con $\mathrm{Si}, \mathrm{Na}, \mathrm{S}, \mathrm{Cl}$ y K. El EDX de la Figura 9B muestra un alto contenido en
$\mathrm{O}=64,13 \%, \mathrm{Ca}=30,12 \%, \mathrm{y}$ $\mathrm{Mg}=3,06 \%$. En menor proporción se encuentran $\mathrm{Na}$, $\mathrm{Si}, \mathrm{S}, \mathrm{Cl}$ y K.

La Figura 10 muestra un zona que parece estar vacía y otra presenta una imagen más clara. El análisis por EDX muestra que la zona vacía presenta un alto contenido en $\mathrm{O}=86,26 \%$ y $\mathrm{Ca}=4,86 \%$ y en menor proporción se detecta $\mathrm{Na}$, $\mathrm{Mg}, \mathrm{Si}, \mathrm{S}, \mathrm{Cl}$ y K. La parte clara de la Figura 10 contiene una concentración de $\mathrm{O}=87,28 \%$ y de $\mathrm{Ca}=$ $4,77 \%$, y en menor concentración los mismos elementos que la zona vacía y en la misma proporción.

La Figura 11 contiene una gran cantidad de cuerpos cristalinos. El análisis por EDX mostró un alto contenido en $\mathrm{O}=70,30 \%$ y en $\mathrm{Ca}=21,6 \%$. En menor proporción se detectó $\mathrm{Na}, \mathrm{Mg}, \mathrm{Si}, \mathrm{S}, \mathrm{Cl}$ y K.

\section{DISCUSIÓN}

El método de elección para el análisis de cálculos puede ser realizado por espectrometría infrarroja por varias razones: se requiere poca cantidad de muestra (del orden de $\mu \mathrm{g}$ ), y el espectro obtenido es totalmente característico para cada uno de los componentes del cálculo, siendo estos espectros como sus huellas dactilares. Así, este método se ha elegido frente al cualitativo clínico ya que este último tiene poca fiabilidad al tratar de caracterizar determinados componentes, como son el oxalato monohidrato y el dihidrato.

Tabla 2. Valoración de porfirinas en enterolitos realizada por HPLC

\begin{tabular}{lc}
\hline Porfirina & g de enterolito \\
\hline Coproporfirnas & $3,4 \mu \mathrm{g}$ \\
Uroporfirinas & $0,34 \mu \mathrm{g}$ \\
Protoporfirinas & $0,2 \mu \mathrm{g}$ \\
Heptacarboxilporfirinas & $0,1 \mu \mathrm{g}$ \\
\hline
\end{tabular}




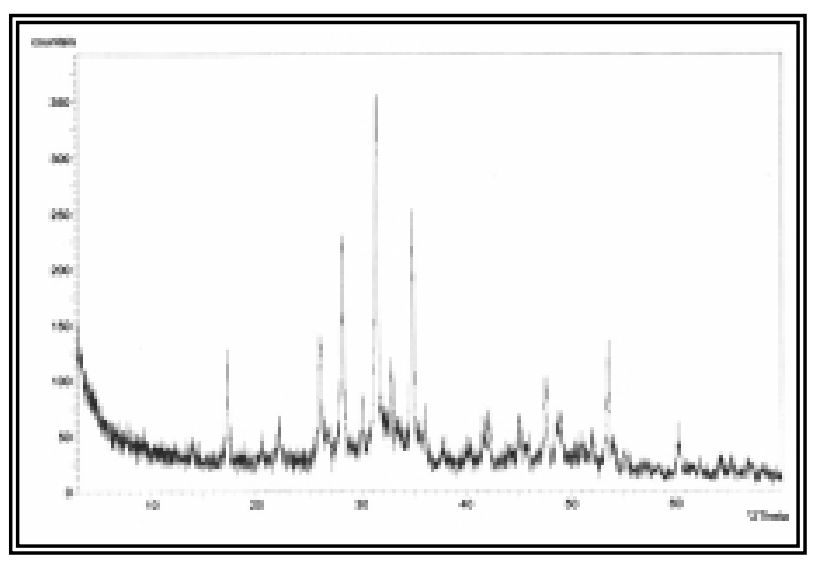

FIGURA 5. Difractograma de un enterolito examinadas bajo microscopia electrónica de barrido (BSE).

Existen otros métodos más sofisticados, y por ello menos disponibles, como la microscopía por corte fino en microscopio con luz polarizada, microscopía electrónica por difracción de rayos $\mathrm{X}$ y la espectrometría de masas. A pesar de todo, a veces es necesario, además de la obtención del
EIR de un cálculo, un método complementario como es el que hemos reflejado en este estudio. Es de destacar que cálculos negruzcos pueden contener porfirinas, lo que hace necesario el estudio de los mismos por análisis por cromatografía en capa fina o por cromatografía líquida de alta resolución.

La peculiaridad de una serie de cálculos que llegaron al laboratorio de Bioquímica de la Fundación Jiménez Díaz-Unilabs nos llevó a la realización de un estudio mas profundo que el rutinario. Estos cálculos eran, generalmente, redondeados, de un color marrón y textura como caramelo que presentaban una superficie con múltiples dibujos realizados por una materia muy oscura, casi negra. Estos cálculos, al cortarlos, estaban vacíos y desde luego, distaban mucho de parecerse a los cálculos renales y vesiculares.

El EIR dio como resultado una composición fundamentalmente de carbonato apatita no-este-
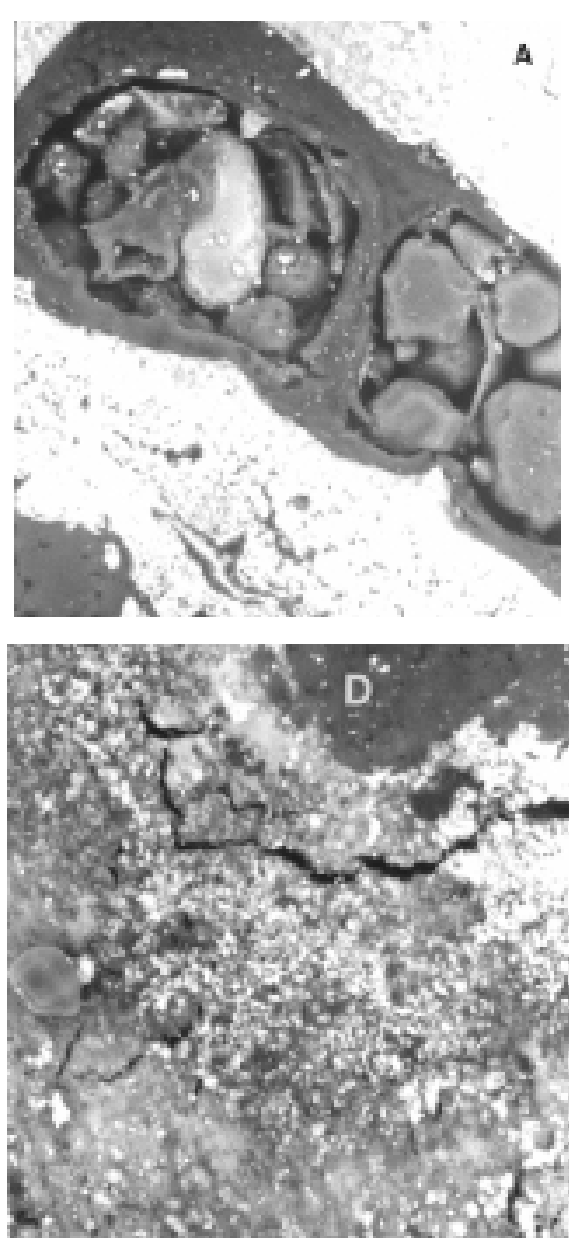
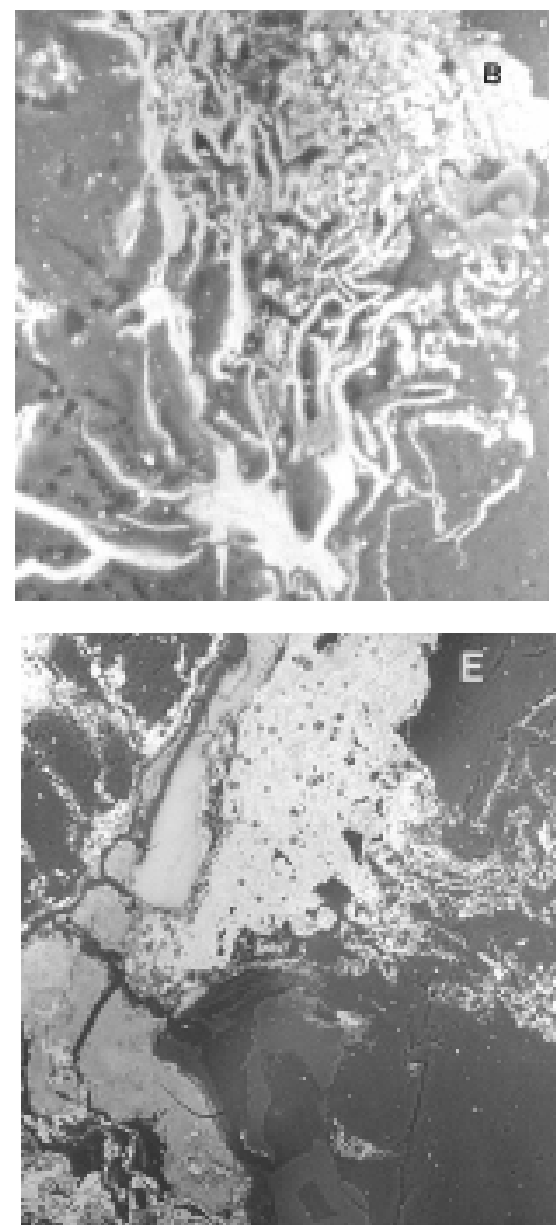

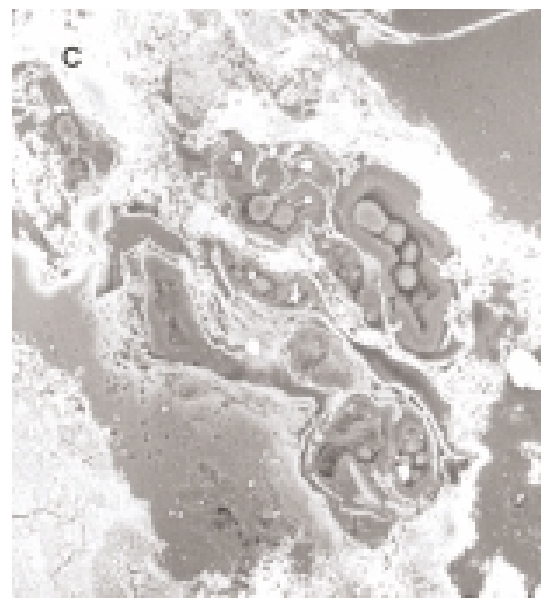

FIGURA 6. Muestras de enterolito exa minadas bajo microscopia electrónica de barrido (BSE). Ver texto. 

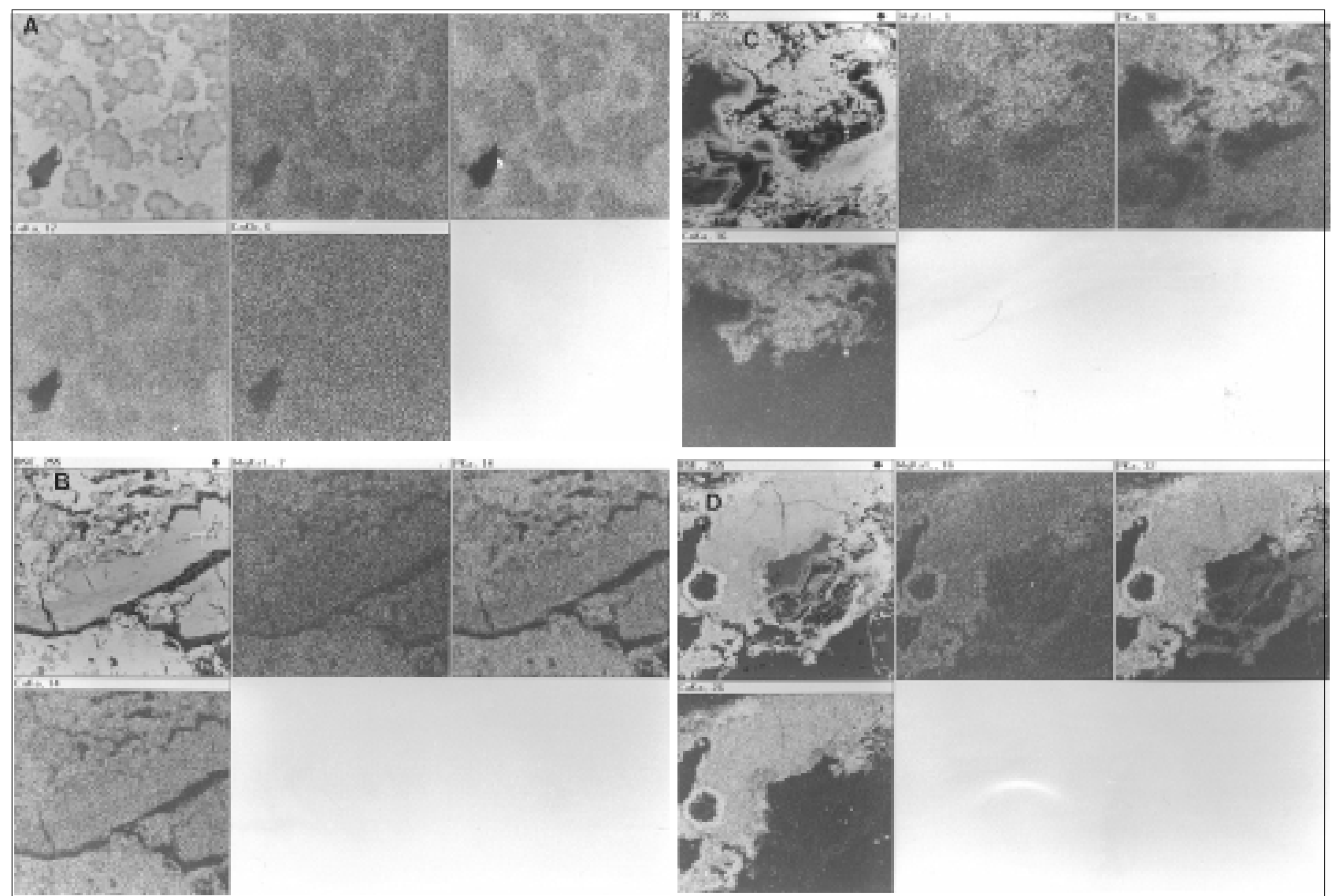

FIGURA 7. Diferentes “mapping” de diversas zonas de un eterolito.

quiométrica, de tipo whitloquita y también se podía detectar la presencia de materia orgánica. En nuestros análisis encontramos que el cálculo contenía porfirinas, destacándose la coproporfirina. El hecho de encontrar porfirinas no es un hecho nuevo ya que en estudios anteriores realizados por nuestro grupo encontramos porfirinas en cálculos negruzcos ${ }^{2,3}$. Estos cálculos presentan dos patrones: en uno aparece un aumento de la coproporfirina y en otro la uroporfirina se encuentra en mayor proporción. Pacientes con hepatopatías eliminan por orina mas coproporfirina, mientras que pacientes con porfiria o fracaso renal eliminan, preferentemente, uroporfirina y hepatoporfirina, es decir, porfirinas mas carboxiladas.

En este caso, se describe que el paciente fue intervenido quirúrgicamente, encontrándose una neoplasia estenosante en el ángulo hepático del colon. Este hecho es importante y la porfirina preferentemente eliminada es la coproporfirina. El caso de haber encontrado por difracción de rayos $\mathrm{X}$ sólo fosfato cálcico $\left(\mathrm{CaP}_{4} \mathrm{O}_{11}\right)$ y no carbonato apatita se puede explicar dado que para que un compuesto pueda ser detectado por difracción de rayos $\mathrm{X}$ tiene que estar perfectamente cristalizado y en nuestro estudio el EIR muestra una carbonato apatita no estequiométrica ${ }^{4}$. Los estudios con EDX en todas las zonas del cálculo analizadas muestran la existencia de los mismos iones, algunos como el $\mathrm{O}, \mathrm{Ca}$. $\mathrm{El} \mathrm{P}$ es lógico encontrarlo ya que se utiliza en la técnica de preparación de la muestra. En nuestro caso era de esperar la existencia de Mg dado que se trata de carbonato apatita de tipo whitloquita (ortofosfato de calcio y magnesio). Un trabajo de GonzálezDíaz y M. Santos ${ }^{5}$ estudia la influencia del Mg en las apatitas. $\mathrm{El} \mathrm{Mg}$ competitivamente sustituye al calcio en la superficie de las apatitas. Además, el $\mathrm{Mg}$ es un inhibidor del crecimiento y formación de cálculos, lo que hace que las apatitas que contengan Mg presenten poca cristalinidad, hecho que corrobora el estudio con difracción de rayos $\mathrm{X}$, en el que no se detectó la carbonato apatita. 

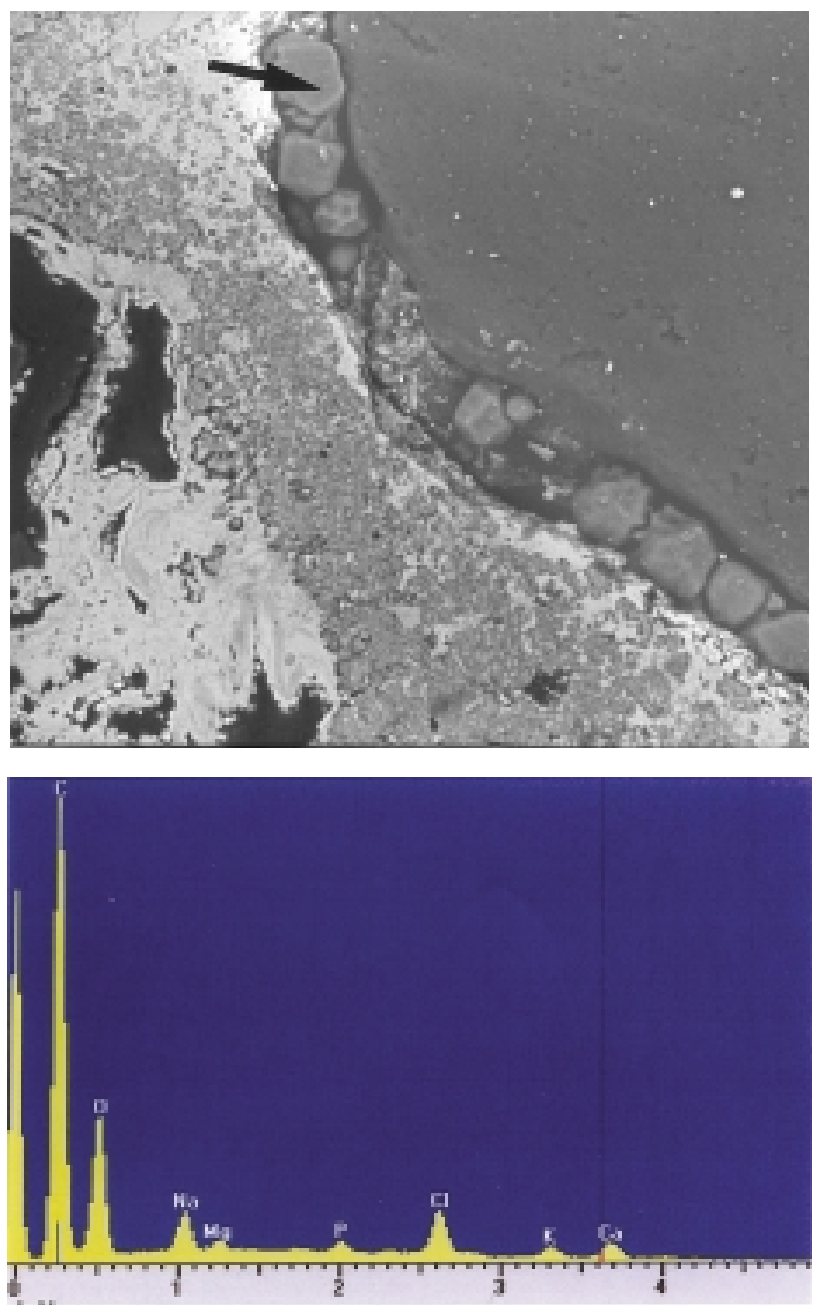

FIGURA 8. Imagen de microscopía electrónica de un enterolito. Espectro por EDX de la figura poliédrica.

Sin embargo, existen otra serie de elementos que hasta el momento no es habitual su presencia, aunque en el trabajo de Rodríguez-Miñón y Cifuentes et al. ${ }^{6}$ se encontraron $\mathrm{Si}, \mathrm{Al}, \mathrm{Fe}, \mathrm{S}$ y Ba y en otro trabajo de nuestro grupo $^{7}$ se encontraron por espectrometría de absorción atómica $\mathrm{Fe}$, $\mathrm{Cu}, \mathrm{Zn}, \mathrm{Mg}, \mathrm{K}$, Na y P. Es curioso comparar que los elementos detectados por EDX y por espectrofotometría de emisión atómica. Aunque ambos métodos detectan $\mathrm{Na}, \mathrm{Ca}, \mathrm{Mg}, \mathrm{K}, \mathrm{Si}, \mathrm{S}, \mathrm{P}, \mathrm{y}$ aunque por espectrofotometría de emisión atómica además detectamos $\mathrm{Zn}, \mathrm{Ba}, \mathrm{Mn}, \mathrm{Fe}, \mathrm{Cu}$, y trazas de Ti, y Br. En EDX el P no se valora ya que el método incluye este elemento.

Ciertos elementos como el $\mathrm{Si}, \mathrm{Al}, \mathrm{S}$ y Fe pueden provenir de medicamentos. Las apatitas pueden también contener $\mathrm{F}, \mathrm{Cl}$ y $\mathrm{Br}$ como sustitu-
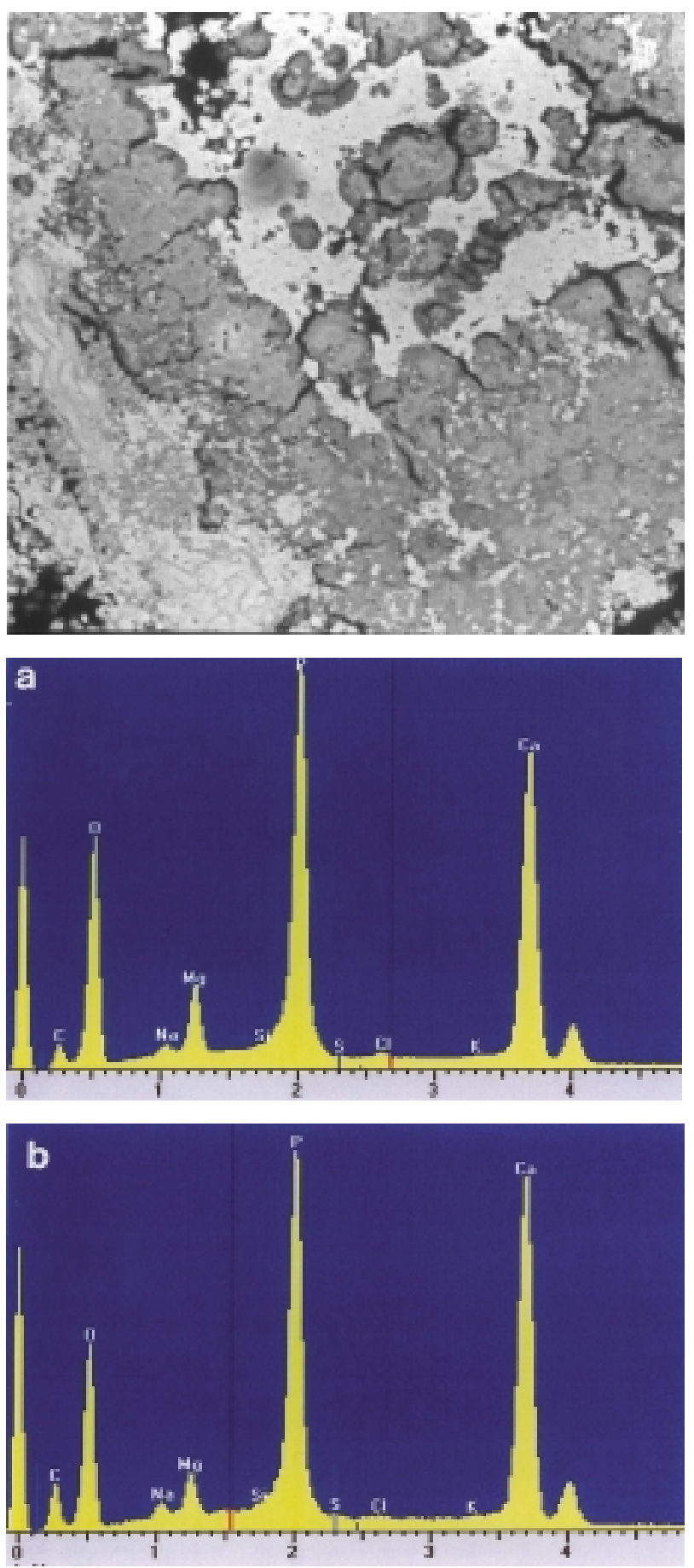

FIGURA 9. Muestra de enterolito examinada bajo microscopia electrónica y espectro EDX de zona clara (a) y de la zona oscura (b).

yente del grupo $\mathrm{OH}$. Nuestro grupo ${ }^{6-8} \mathrm{y}$ Medina y cols $^{9}$ encontraron un cálculo de sílice, debido a tratamiento farmacológico. Nuestro grupo también encontró un cálculo que contenía $\mathrm{SO}_{4} \mathrm{Ca}$ - 

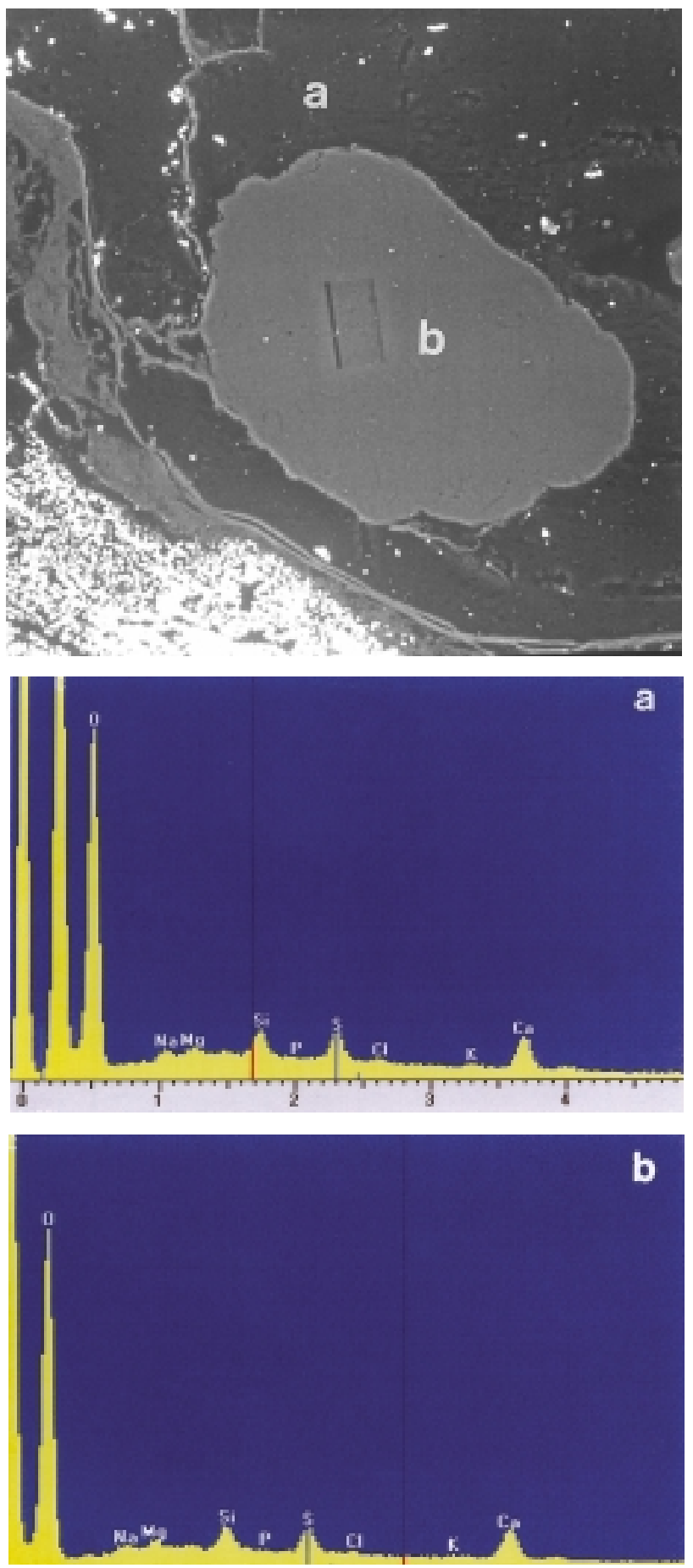

FIGURA 10. Imagen por microscopia electrónica (a) y espectro por EDX (b) de un enterolito.

$2 \mathrm{H}_{2} \mathrm{O}$ por EIR y microscopía electrónica de barrido (MEB) y por $\mathrm{EDX}^{10}$.

Este trabajo muestra un detenido y profundo estudio de enterolitos intestinales, hecho inusual en humanos, que proceden de un paciente de 91
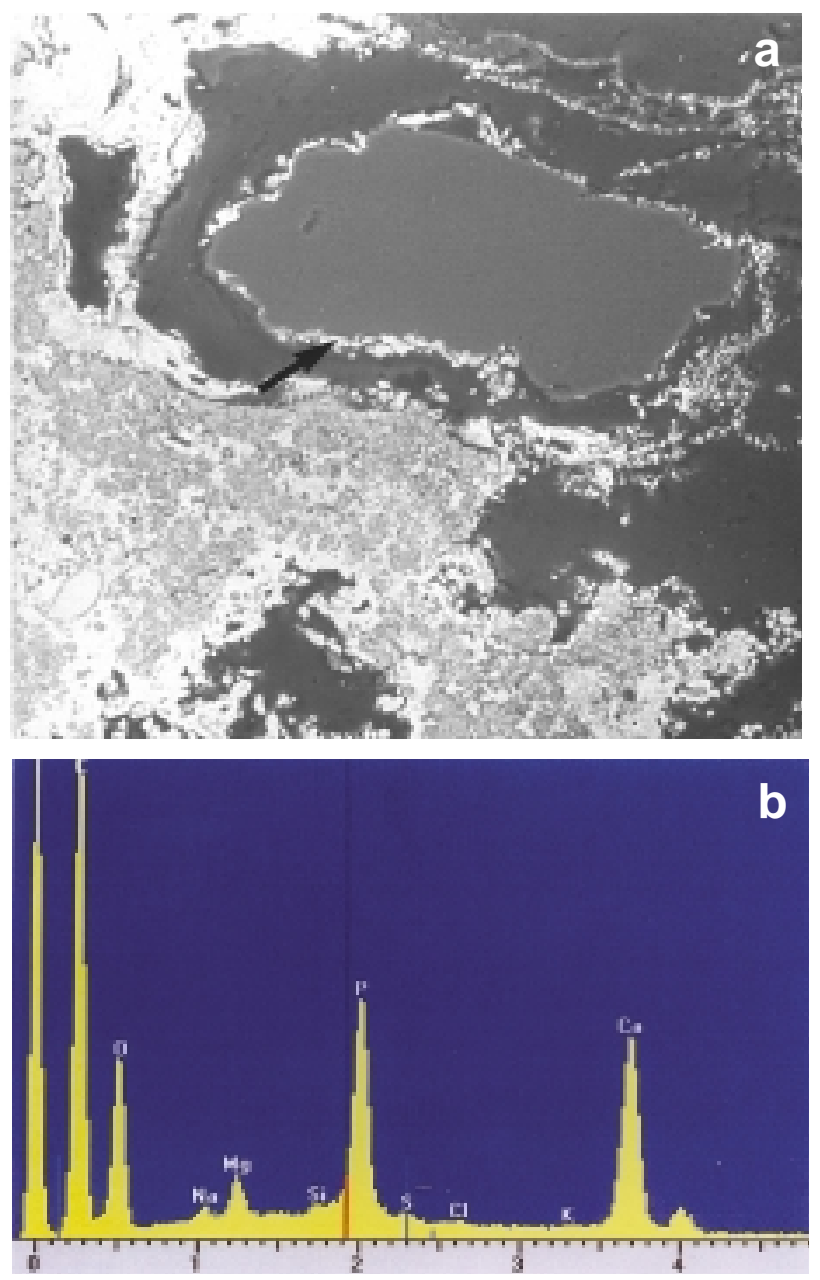

FIGURA 11. Imagen por microscopia electrónica (a) y espectro por EDX (b) de un enterolito.

años afectado de adenocarcinoma de colon. De los análisis realizados podemos deducir que los componentes encontrados en el cálculo intestinal son similares a los encontrados en la litiasis renal aunque su morfología dista mucho de la presentada en cálculos renales.

Agradecimientos. A la Dra C Ascaso y T. GarcíaGonzález por su colaboración en los estudios con microscopía electrónica y EDX y difracción de rayos $\mathrm{X}$ respectivamente (CSIC, Madrid).

\section{REFERENCIAS}

1. Ascaso C, Wierzchos J. New applications of submicroscopic techniques in the study of biodegradation caused by lichen thalli. Microbiologia 1994;10(1-2):103-110.

2. Traba ML, Perpiña J, Enríquez de Salamanca R. Identification and cuantification of various porphyrins as components in a peculiar type of renal calculus. Arch Esp Urol 1985;38:601-604. 
3. Traba ML, Perpiña J, Enriquez de Salamanca R, Rapado A. Porphyrin accumulation in some types of renal calculi. Med Sci Res 1988;16:1269-1271.

4. González-Díaz PF, García-Ramos JV, Santos M. Compositium of apatites in human urinary calculi. Calcif Tissue Int 1979;28:215-225.

5. González-Díaz PF, Santos M. Magnesium influence in calcium apatites from renal calculi. In: Pathogenese und Klinik der Harnsteiner VI (Bonn, 1978, pg 340).

6. Rodríguez-Miñón JL, Salvador E, Bellanato J, Medina JA. Silica, Aluminium iron, sulfur and barium in a urinary calculus. Actas Urol Esp 1994;18(supp):452-454.

7. Traba ML, Arroyo F, Rubio M, Nuncio J, Perpiña J, Enriquez de Salamanca E. Analysis of ionic components, proteins and lipids in calculi containing porphyrins. Actas Urol Esp 1987; 9:307-308.

8. Rapado A, Traba ML, Caycho C, Cifuentes-Delatte L. Druginduced renal stones: incidence, clinical expression and stone analysis. Contrib Nephrol 1987;58:25-29.
9. Medina SA, Rodríguez J, Santos M, Cifuentes-Delatte L. Siliceous renal calculi in a man (11th case in the world). Rev Clin Esp 1978;151(5):411-413.

10. Cifuentes-Delatte L, Medina JA, Traba ML. A calcium sulfate calculus of renal origin. A verifiable observation?. Arch Esp Urol 1983;36:117-123.

Dra. Ma Luisa Traba Villameytide

Laboratorio de Bioquímica.

Sección de Fisiopatología Ósea

Fundación Jiménez Díaz-UTE

Avda. Reyes Católicos, 2

28040 Madrid

(Trabajo recibido el 10 de mayo de 2005) 